\title{
EPCR promotes MGC803 human gastric cancer cell tumor angiogenesis in vitro through activating ERK1/2 and AKT in a PAR1-dependent manner
}

\author{
QINGLING WANG ${ }^{1,2}$, YANGYANG TANG ${ }^{1,2}$, TIANYUAN WANG ${ }^{1,2}$, HONG-LI YANG $^{1,2}$, \\ XINYUE WANG ${ }^{3}$, HONGRU MA ${ }^{4}$ and PENG ZHANG ${ }^{2,5}$ \\ ${ }^{1}$ Department of Pathology, Xuzhou Medical University; ${ }^{2}$ Jiangsu Key Laboratory of Immunity and Metabolism; \\ Schools of ${ }^{3}$ Anesthesiology and ${ }^{4}$ Clinical Medicine; ${ }^{5}$ Department of Pathogenic Biology and Immunology, \\ Xuzhou Medical University, Xuzhou, Jiangsu 221004, P.R. China
}

Received December 5, 2017; Accepted May 22, 2018

DOI: $10.3892 / \mathrm{ol} .2018 .8869$

\begin{abstract}
The endothelial cell protein C receptor (EPCR) serves a key role in activated protein C (APC)-mediated cytoprotective effects in endothelial cells, and is involved in the development of certain types of human cancer. To the best of our knowledge, the present study is the first to demonstrate that EPCR may exert effects on gastric cancer angiogenesis in vitro. To detect microvessel density (MVD), the microvascular endothelial cells were stained for cluster of differentiation (CD)31 and CD34 in 61 cases of surgical resection of gastric carcinoma tissues, and the association between the expression of EPCR protein and MVD was analyzed. In addition, to analyze the effect of EPCR expressed by gastric cancer cells on the proliferation, migration and angiogenic abilities of endothelial cells, human umbilical vein endothelial cells (HUVECs) were cultured with tumor-conditioned medium derived from EPCR knockdown or protease-activated receptor 1 (PAR1)-blocked MGC803 gastric cancer cells. A CCK- 8 assay was used to assess the proliferation ability of the HUVECs. A Transwell assay was performed to assess the migration ability of the HUVECs and a Matrigel-based tube formation assay was used to assess the angiogenic activity of the HUVECs. The results demonstrated that the expression of EPCR was correlated with the MVD of gastric cancer tissues. When cultured with tumor-conditioned medium derived from EPCR knockdown or PAR1-blocked
\end{abstract}

Correspondence to: Dr Peng Zhang, Department of Pathogenic Biology and Immunology, Xuzhou Medical University and Jiangsu Key Laboratory of Immunity and Metabolism, 209 Tongshan Road, Xuzhou, Jiangsu 221004, P.R. China

E-mail: pengzh@xzhmu.edu.cn

Abbreviations: EPCR, endothelial protein C receptor; PAR1, proteaseactivated receptor 1; MVD, microvessel density; HUVECs, human umbilical vein endothelial cells

Key words: gastric cancer, tumor angiogenesis, endothelial protein $\mathrm{C}$ receptor, protease-activated receptor 1 , extracellular signal regulated kinase $1 / 2$, protein kinase $B$
MGC803 cells, the proliferation, migration and tubules formation abilities of HUVECs were markedly inhibited markedly. The expression of phosphorylated (p)-extracellular signal regulated kinase 1/2, p-protein kinase B (AKT; s473) and p-AKT (T308) in the HUVECs was decreased. In addition, EPCR knockdown inhibited PAR1 activation in the MGC803 cells. These results indicated that the expression of EPCR in gastric cancer cell line MGC803 contributes to tumor angiogenesis in vitro by activating ERK1/2 and AKT, and that this effect of EPCR is dependent on PAR1 activation.

\section{Introduction}

Angiogenesis is a vital process in the growth, development and spread of tumors. It is a multi-step process that is determined by a net balance between pro- and antiangiogenesis regulators. At present, anti-angiogenesis is regarded as a target for cancer therapy (1).

Human endothelial cell protein C receptor (EPCR) is a type 1 transmembrane glycoprotein that is expressed primarily by the vascular endothelium of larger blood vessels $(2,3)$. On the endothelial surface, EPCR binds and presents protein C (PC) to the thrombin:thrombomodulin (TM) complex to generate activated protein C (APC). It binds both PC and APC with equally high affinity (4). APC is critical for the negative regulation of blood coagulation by inactivating two key cofactors FVIIIa and FVa, which are responsible for amplification of blood coagulation reactions, and which promote thrombin generation (4). APC plays a cytoprotective role in endothelial tissue, which involves altering gene expression profiles, anti-apoptotic activity, anti-inflammatory activity and protection of endothelial barriers $(2,5)$. This cytoprotective effect of APC requires EPCR and the protease activated receptor 1 (PAR1). In addition, APC can induce endothelial cell proliferation and angiogenesis by activating mitogen-activated protein kinase (MAPK) (6). Recent research showed that EPCR is expressed in tumor cells, including leukemia U937 cells (7), mesothelioma (8), ovarian cancer (9), lung cancer $(10,11)$ and breast cancer cells $(12,13)$. In our previous study, we had found that EPCR is expressed in gastric carcinoma tissue 
and BGC803, HGC27, AGS and SGC7901 gastric carcinoma cancer cells, and that it can promote MGC803 gastric cancer cells proliferation and migration (14). However the role of EPCR in tumor angiogenesis is not clear.

In this study, we investigated the correlation between the expression of EPCR and the microvessel density (MVD) of tumors in the primary respectable gastric carcinoma, through quantification of MVD using the specific endothelial cell markers CD31 and CD34. From this, we observed that the mean MVD value was higher in EPCR-positive gastric cancer samples compared with that in negative samples. Additionally, the proliferation, migration and tubule formation of human umbilical vein endothelial cells (HUVECs), when cultured with the tumor-conditioned medium of MGC803 cells treated with PAR1 antibody or subject to EPCR knockdown, were inhibited. These findings indicate a novel role of EPCR in gastric cancer progression.

\section{Materials and methods}

Antibodies. Mouse monoclonal anti-EPCR (ab151403; Abcam, Cambridge, MA, USA), rabbit polyclonal anti-PAR1 (ab63445; Abcam, Cambridge, UK), rabbit monoclonal anti-pERK1/2 (4370), anti-pAKT (Ser473) (4060), anti-pAKT (Thr308) (13038), and anti-AKT (9272) (all from Cell Signaling Technology, Danvers, MA, USA), rabbit polyclonal anti-ERK1/2 (c0185; Anbo Biotech Co., Ltd., San Francisco, CA, USA), mouse monoclonal anti-GAPDH (TA505454; Zhongshan Biotech Co.,Ltd., Beijing, China), rabbit anti-mouse (ab6728) and goat anti-rabbit (ab6721) (both from Abcam, Cambridge, UK) were used in the present study.

Cell culture. Human gastric cancer cell line MGC803 and HUVECs, were purchased from the Shanghai Institute of Biochemistry and Cell Biology, Chinese Academy of Sciences (Shanghai, China). Cells were cultured in Dulbecco's modified Eagle's medium (Gibco; Thermo Fisher Scientific, Inc., Waltham, MA, USA) supplemented with $10 \%$ fetal bovine serum (FBS; HyClone, Logan, UT, USA), $100 \mathrm{IU} / \mathrm{ml}$ penicillin and $100 \mathrm{ng} / \mathrm{ml}$ streptomycin in a $37^{\circ} \mathrm{C}, 5 \% \mathrm{CO}_{2}$ humidified atmosphere.

siRNA transfection. Stealth ${ }^{\mathrm{TM}}$ RNA duplexes against human EPCR (sense, 5'-GCACUCGGUAUGAACUGCGGGAAUU-3' and antisense, 5'-AAUUCCCGCAGUUCAUACCGAGUGC-3') were designed and synthesized as previously described (14). The anti-EPCR siRNA (50 $\mathrm{nM})$ was transfected using Lipofectamine 2000 (Invitrogen; Thermo Fisher Scientific, Inc.) according to the manufacturer's protocol.

Immunohistochemical analysis of CD31 and CD34. Section of a tissue microarray with 61 gastric carcinoma tissues collected from the Affiliated Hospital of Xuzhou Medical University (14) were incubated at $4^{\circ} \mathrm{C}$ overnight in a moist chamber with mouse monoclonal anti-CD31 and CD34 antibodies (Maxim-Bio Ltd., Fuzhou, China). The sections were then incubated at room temperature for $10 \mathrm{~min}$ with biotinylated anti-mouse immunoglobulin after being washed with $0.02 \mathrm{M}$ phosphate buffered saline (PBS) pH 7.4. Then, the sections were incubated for 10 min with Streptavidin-Biotin Complex (Boster Ltd., Wuhan, China) after being washed with PBS. CD31 and
CD34 labeling was visualized by incubating the sections in DAB solution (Boster Ltd.). MVD was assessed by counting the vessel numbers under three different fields at high-power magnification fields (x400). An average was calculated for each case and statistically presented as the mean $\pm \mathrm{SD}$. The isolated immuno-reactive endothelial cells or groups of endothelial cells separated by the adjacent microvessels were considered to be quantifiable individual vessels. Visible lumens or the presence of associated red cells were not obligatory.

Preparation of tumor-conditioned medium.Tumor-conditioned medium was prepared as described in previous studies (15-20). In brief, after MGC803 cells were treated with $10 \mu \mathrm{g} / \mathrm{ml}$ anti-PAR1 antibody or transfected with EPCR siRNA, the culture medium was collected and centrifuged, and the supernatant was collected. The supernatant was combined with fresh DMEM according to a ratio of fresh DMEM:FBS:Tumor cell culture medium of 5:1:4, to obtain the tumor-conditioned medium. Subsequently, the HUVECs were cultured with the prepared tumor-conditioned medium.

Proliferation analysis. A total of $3 \times 10^{3}$ HUVECs were plated in 96-well plates in the tumor-conditioned medium, and cultured for 24, 48 and $72 \mathrm{~h}$, respectively. Then, $10 \mu \mathrm{l}$ WST-8 [2-(2-methoxy4-nitrophenyl)-3-(4-nitrophenyl)-5-(2,4-disulfophenyl)-2H-tetrazolium, monosodium] from a CCK-8 kit was added to each well and incubated for $4 \mathrm{~h}$. The absorbance was measured at $450 \mathrm{~nm}$ on a Muti-Detection Microplate Reader (Thermo 1500; Thermo Fisher Scientific, Inc.).

Transwell assay. The migration ability of HUVECs was examined using a Transwell cell culture chamber (Corning Incorporated, Corning, NY, USA). The lower chamber was filled with the prepared tumor-conditioned medium $(600 \mu \mathrm{l})$, and $1 \times 10^{4}$ cells were seeded onto the upper chamber. Chambers were incubated for $24 \mathrm{~h}$ at $37^{\circ} \mathrm{C}$. The cells remaining on the top surface of the membrane were removed with application of a cotton swab followed by washing with PBS three times. The cells on the bottom surface of the membrane were fixed and stained with $0.1 \%$ crystal violet. Subsequently, the number of migrated cells was quantified by counting in 5 fields of view under a light microscope (x20 objective).

Matrigel-based tube formation assay. Matrigel(BD Biosciences, San Jose, CA, USA) was plated into 96-well plates at $50 \mu \mathrm{l} /$ well and incubated for $30 \mathrm{~min}$ at $37^{\circ} \mathrm{C}$. Then, $2 \times 10^{4}$ HUVECs were re-suspended with tumor-conditioned medium, seeded onto the Matrigel, and incubated overnight at $37^{\circ} \mathrm{C}$, Each well was analyzed directly under a microscope, and tubules from 3-5 random fields of each well were imaged and counted.

Western blot analysis. After treatment with the prepared tumor-conditioned medium, HUVECs were lysed with RIPA buffer. Cell lysates (30 $\mu \mathrm{g} /$ lane) were subjected to 15\% SDS-PAGE and transferred onto polyvinylidene difluoride membranes (EMD Millipore, Billerica, MA, USA). After blocking in PBST $(10 \mathrm{mmol} / \mathrm{l}$ Tris- $\mathrm{HCl}, \mathrm{pH} 7.4,150 \mathrm{mmol} / \mathrm{l}$ $\mathrm{NaCl}, 0.05 \%$ Tween-20) containing 5\% nonfat dried milk for $1 \mathrm{~h}$, the membranes were incubated with the mouse monoclonal anti-EPCR $(1: 2,000)$, rabbit polyclonal anti-PAR1 
$(1: 2,000)$, rabbit monoclonal anti-pERK1/2 (1:3,000), anti-pAKT (Ser473) (1:3,000), anti-pAKT (Thr308) $(1: 3,000)$, anti-AKT $(1: 3,000)$, rabbit polyclonal anti-ERK1/2 $(1: 3,000)$, and mouse monoclonal anti-GAPDH $(1: 1,000)$ at $4^{\circ} \mathrm{C}$ overnight. Secondary antibodies $(1: 10,000)$ were incubated at room temperature for $1 \mathrm{~h}$. Protein bands were detected by the enhanced chemiluminescence (ECL) reaction (Bio-Rad Laboratories, Inc., Hercules, CA, USA).

Cell ELISA. ELISA was performed as described previously (21). Briefly, $3 \times 10^{4}$ cells were seeded in a flat-bottomed 96-well microtiter plate and incubated for $24 \mathrm{~h}$ at $37^{\circ} \mathrm{C}$ in $5 \% \mathrm{CO}_{2}$. Cells were washed 3 times with PBS and fixed with $100 \mu \mathrm{l}$ of $4 \%$ paraformaldehyde solution in $0.01 \mathrm{M}$ PBS for $10 \mathrm{~min}$. Then, cells were incubated with $100 \mu 1$ of a blocking solution containing $1 \%(\mathrm{w} / \mathrm{v}) \mathrm{BSA}$ in $0.01 \mathrm{M}$ PBS for $1 \mathrm{~h}$. After blocking, $50 \mu \mathrm{l} /$ well of the primary antibody (anti-uncleaved PAR1 antibody was designed and prepared by Abgent Biotechnology Co., Ltd., Suzhou, China, peptide: NH2-SFLLRNPNDKC-CONH2; peptide control, NH2-DPRSFLLRNPNDKC-CONH2) or $50 \mu \mathrm{l} /$ well of the blocking solution was added and incubated for at least $1 \mathrm{~h}$ at $4^{\circ} \mathrm{C}$. After washing 5 times with $200 \mu \mathrm{l} /$ well of the washing buffer, $50 \mu \mathrm{l} /$ well of the secondary antibody or $50 \mu \mathrm{l} /$ well of the blocking solution was added and incubated for an additional $1 \mathrm{~h}$ at $4^{\circ} \mathrm{C}$. Then, $100 \mu \mathrm{l}$ of 3,3', 5,5'-tetramethylbenzidin e (TMB) substrate solution was added to each well after washing 5 times, and incubated for $20 \mathrm{~min}$ at room temperature. Finally, $25 \mu \mathrm{l} /$ well of $2 \mathrm{M}$ sulfuric acid was added to stop the enzyme reaction, and absorbance was measured at $450 \mathrm{~nm}$ using muti-detection microplate reader (Thermo 1500).

Statistical analysis. All statistical analyses were performed using SPSS software (version 16.0; SPSS, Inc., Chicago, IL, USA). Measurement data were representative of experiments repeated at least three times and presented as mean \pm SEM. The results were analyzed among groups using ANOVA followed by the post-hoc Student-Newman-Keuls procedure for multiple comparisons. The Student's paired t-test was used to assess the significance of data comparisons between two groups. P-values of less than 0.05 were considered statistically significant $(\mathrm{P}<0.05)$. Correlations between qualitative data and quantitative data were analyzed with the Eta value, with $\alpha=0.05$ as the inspection level.

\section{Results}

Correlation between EPCR expressions and MVD in gastric carcinoma. In previous study, we had found EPCR to be highly expressed in tissue samples of 44/61 (72.13\%) cases of gastric carcinoma (14). In the current study, the MVD value of these 61 cases of gastric carcinoma was determined by detecting the microvascular endothelial cells markers CD31 and CD34 through immunohistochemistry (Fig. 1). Then, the correlation between the expression of EPCR protein and MVD was analyzed by statistical analysis. As shown in Table I, we observed that the mean MVD value was higher in EPCR-positive gastric cancer samples compared with that in negative samples; and this association was statistically significant.
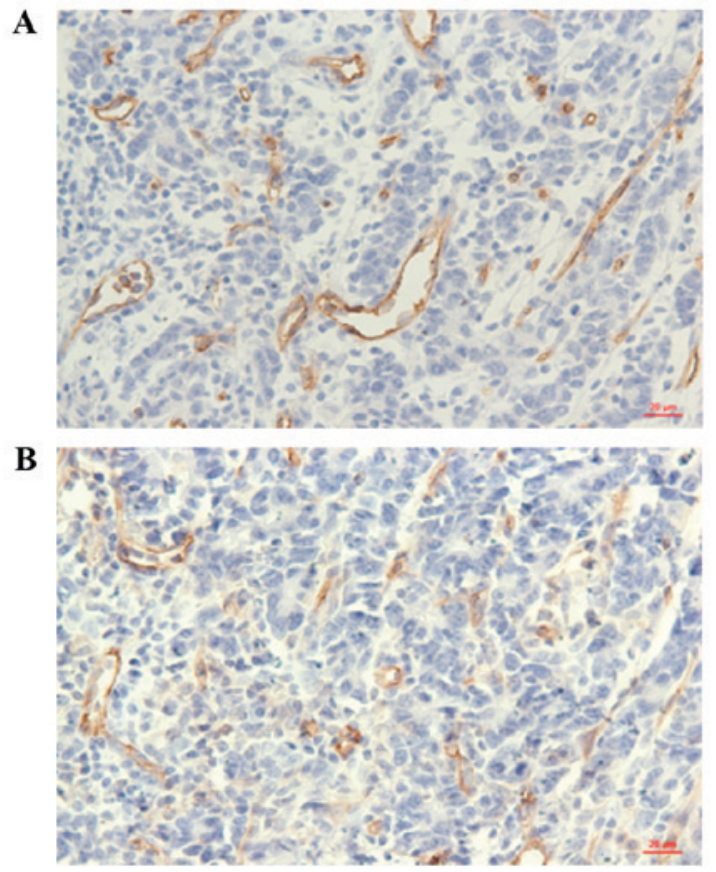

Figure 1. CD31 and CD34 expression in gastric carcinoma. Representative immunohistochemical images of (A) CD34 and (B) CD31 labeling in the same human gastric adenocarcinoma sample. Scale bar, $20 \mu \mathrm{m}$. CD, cluster of differentiation.

Knockdown of EPCR or blockade of PARl inhibits HUVECs growth, migration and tubules formation. In our previous study, we found that EPCR knockdown inhibited the cells growth and migration of MGC803 cells, and that the role of EPCR may be related to PAR1 (14). To study whether knockdown of EPCR and blockade of PAR1 in MGC803 cells affects tumor angiogenesis, tumor-conditioned medium was prepared after MGC803 cells were transfected with EPCR siRNA or treated with $10 \mu \mathrm{g} / \mathrm{ml}$ anti-PAR1 antibody, and then used to culture HUVECs. Then, the cell viability, migration, and tubule formation abilities of the HUVECs were detected. Compared with the control group, the cell viability (Fig. 2A), migrated cell number (Fig. 2B and C), and tubules number (Fig. 2D and E) of the EPCR siRNA-treated group and anti-PAR1 antibody treated-group were decreased significantly. Additionally, compared with the EPCR siRNA group, the cell viability, migrated cell number, and tubule number of the anti-PAR1 antibody-treated group was decreased significantly.

Knockdown of EPCR inhibits activation of PARI in MGC803 cells. To study whether the EPCR expression in MGC803 cells affect tumor angiogenesis through activating PAR1, following EPCR knockdown, anti-uncleaved PAR1 antibody was used to detect the uncleaved PAR1 on the cell membrane of MGC803 cells by Cell ELISA. The results showed that PAR1 protein expression level did not changed after EPCR knockdown (Fig. 3A). However, anti-uncleaved PAR1 antibody-binding rate was increased after EPCR knockdown, compared with a control group and positive control group treated with thrombin, as a known activator of PAR1. Additionally, the anti-uncleaved PAR1 antibody-binding rate of the thrombin-treated group was decreased compared with the control (Fig. 3B). These results 
Table I. Association between EPCR expression and MVD in gastric carcinoma.

\begin{tabular}{cccccccr}
\hline EPCR & $\mathrm{n}$ & MVD CD34 (mean $\pm \mathrm{SD})$ & Eta & P-value & MVD CD31(mean \pm SD) & Eta & P-value \\
\hline+ & 44 & $49.523 \pm 19.471$ & 0.309 & $<0.05$ & $37.899 \pm 20.644$ & 0.427 & $<0.001$ \\
- & 17 & $36.042 \pm 17.391$ & & & $19.421 \pm 5.185$ & & \\
\hline
\end{tabular}

Correlations between qualitative data and quantitative data were analyzed using the Eta value, with $\alpha=0.05$ as the inspection level. EPCR, endothelial cell protein $\mathrm{C}$ receptor; MVD, microvessel density; CD, cluster of differentiation.
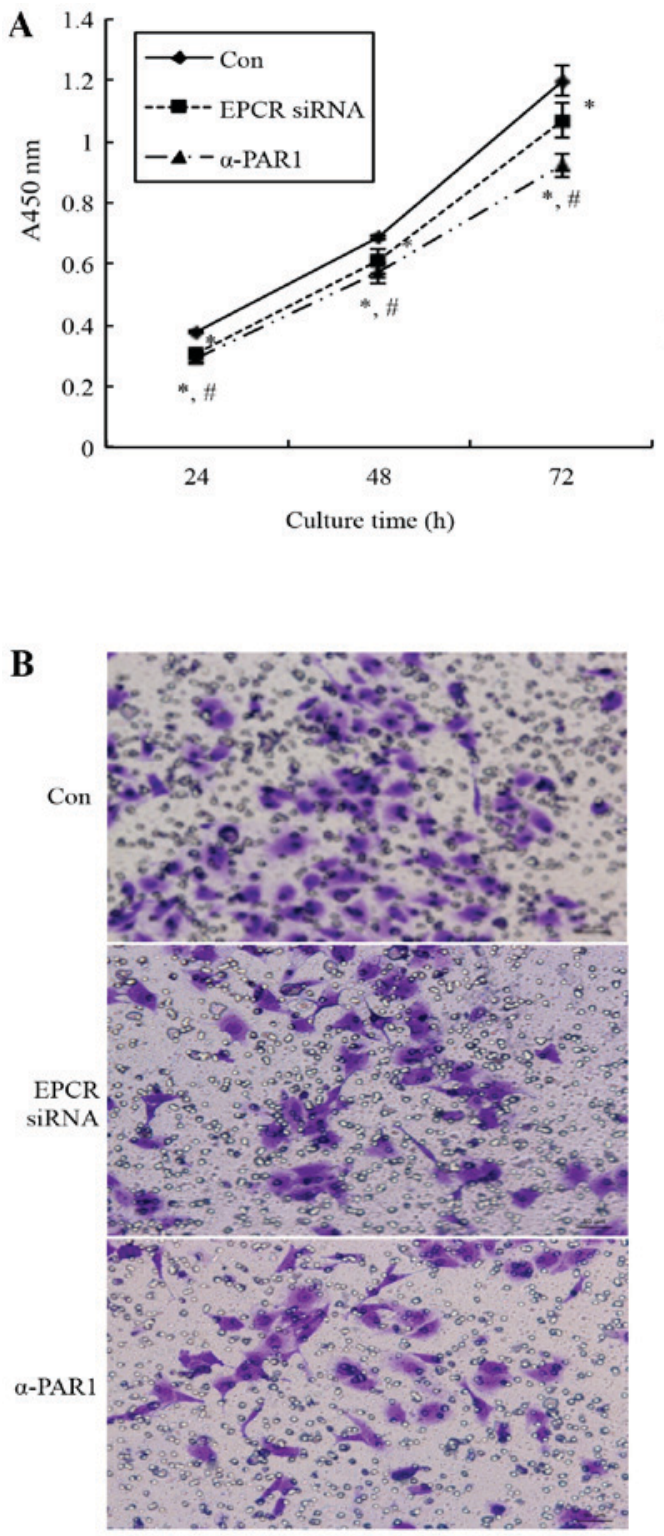
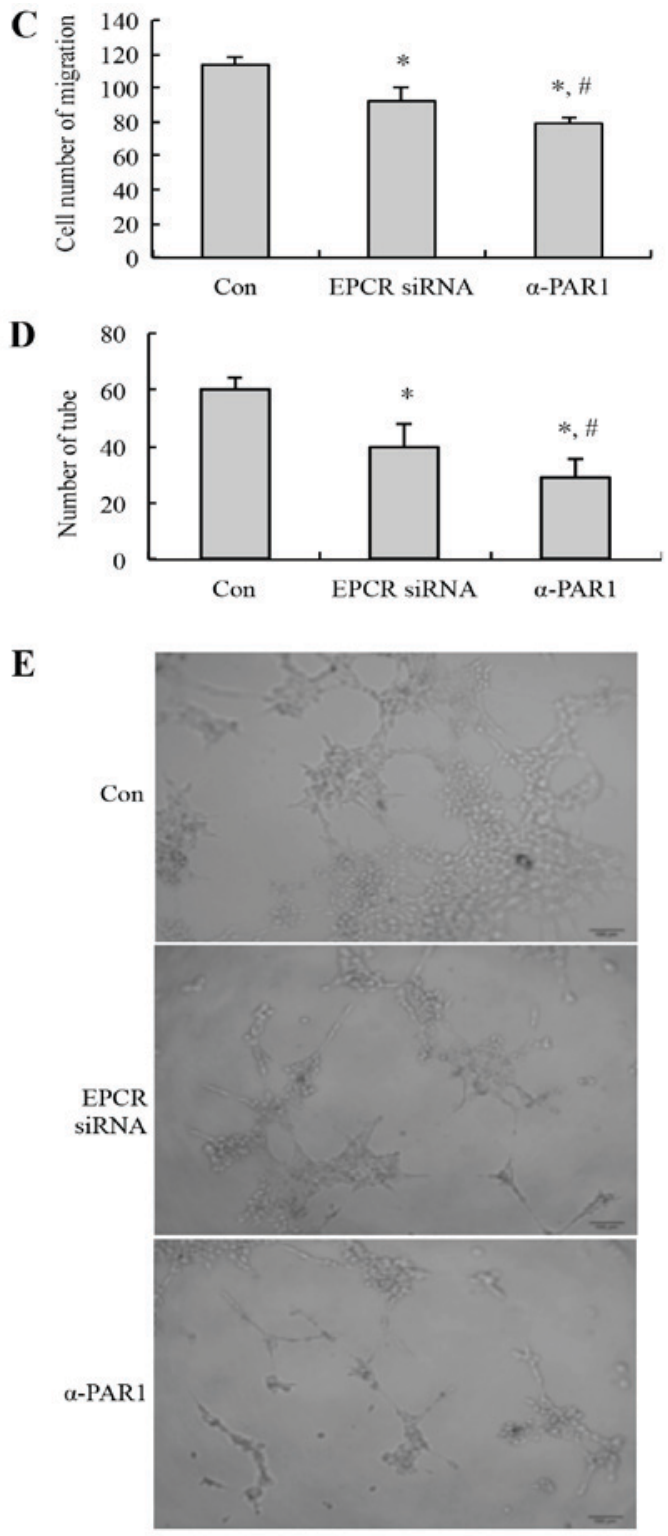

Figure 2. Tumor-conditioned medium of MGC803 cells treated with EPCR siRNA or PAR1 antibody inhibits HUVECs proliferation, migration and tubule formation. (A) Cell viability was detected by cell counting kit- 8 assay. (B and C) Cell migration ability was detected by Transwell assay. Scale bar, $50 \mu \mathrm{m}$. (D and E) Matrigel-based tube formation assay. Scale bar, $100 \mu \mathrm{m}$. All results are presented as the means \pm standard deviation. "P<0.05 vs. control; ${ }^{\sharp} \mathrm{P}<0.05$ vs. EPCR siRNA group. Si, short interfering; con, control; EPCR, endothelial cell protein C receptor; PAR1, protease-activated receptor 1.

indicate that the knockdown of EPCR inhibited PAR1 activation in MGC803 cells.

Knockdown of EPCR or blockade of PAR1 inhibist ERK1/2 and AKT activation in HUVECs. After treatment with tumor-conditioned medium, compared with the control group, the levels of
HUVEC's ERK1/2, AKT (S473) and AKT (Th308) phosphorylation in HUVECs in the EPCR siRNA-treated group and anti-PAR1 antibody-treated group were reduced (Fig. 4). This result indicates that EPCR and PAR1 may promote HUVECs proliferation and migration through ERK1/2 and AKT activation in MGC803 gastric cancer cells. 
A

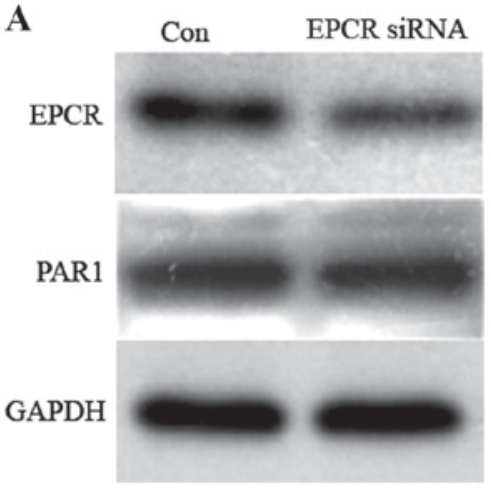

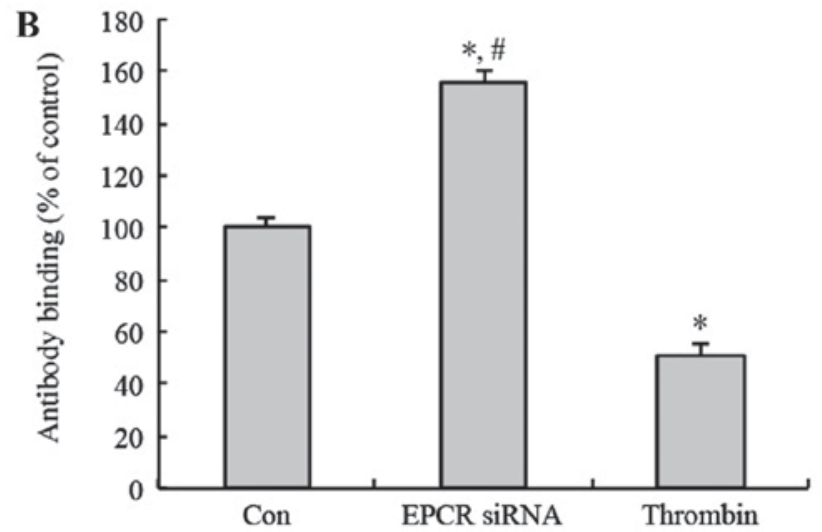

Figure 3. Knockdown of EPCR inhibits activation of PAR1 in MGC803 cells. (A) The protein expression of EPCR and PAR1 was detected by western blotting. (B) The anti-uncleaved PAR1 antibody-binding rate was detected by ELISA assay. All results are presented as the means \pm standard deviation. ${ }^{*} \mathrm{P}<0.05$ vs. control; ${ }^{\mathrm{P}}<0.05$ vs. thrombin-treated group. EPCR, endothelial cell protein $\mathrm{C}$ receptor; PAR1, protease-activated receptor 1.

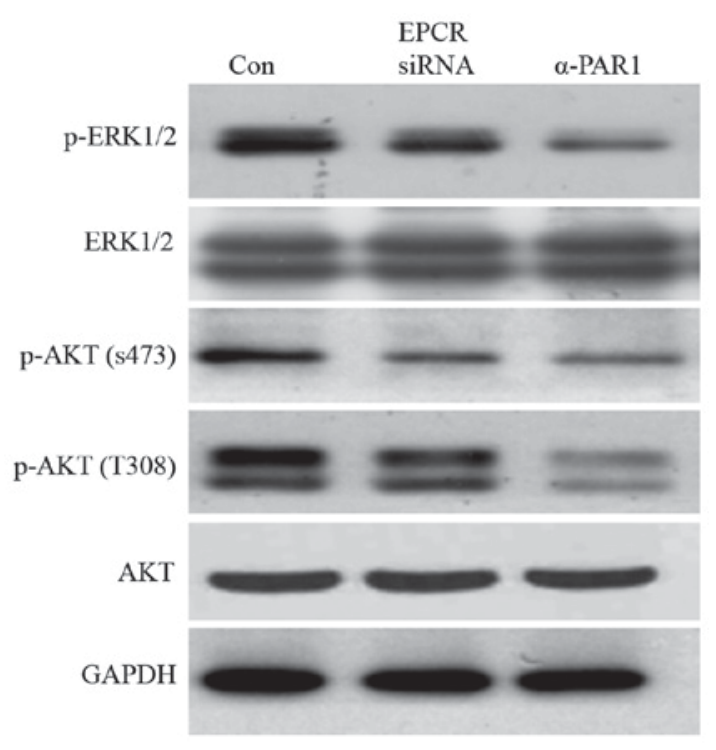

Figure 4.Expression level of p-ERK1/2,p-AKT (Ser473) and p-AKT (Thr308) in HUVECs after treatment with tumor-conditioned medium was detected by western blotting. AKT, protein kinase B; p-, phosphorylated; ERK, extracellular signal regulated kinase; HUVEC, human umbilical vein endothelial cell; EPCR, endothelial cell protein C receptor; PAR1, protease-activated receptor 1 ; si, short interfering.

\section{Discussion}

In the present study, we showed that the expression of EPCR is correlated with MVD in gastric cancer tissues. Furthermore, when cultured with tumor-conditioned medium of gastric cancer MGC803 cells treated with EPCR siRNA or blocking antibodies against PAR1, the proliferation, migration and tubules formation abilities, and the phosphorylation levels of ERK and AKT in HUVECs were decreased. PAR1 activation in MGC803 cells was also decreased after EPCR knockdown.

It has been reported that APC induces HUVEC proliferation, morphogenetic changes resembling tube-like structures, and angiogenesis in the mouse cornea; while antibodies against EPCR inhibit HUVEC proliferation (6). Niessen et al found that EPCR/APC-PAR1 signaling prevented inflammation-induced vascular leakage, and pharmacological or genetic blockade of this pathway leaded to mice sensitivity to LPS-induced lethality in mice (22). Sundaram et al found FVIIa could reduce LPS-induced vascular leakage in the lung and kidney; but the protective effect was attenuated in EPCR-deficient mice, and blocked by PAR1. In addition they found VEGF-induced vascular leakage in the skin was highly dependent on EPCR expression levels (23). Mosnier and Griffin found that APC could inhibit staurosporine-induced apoptosis of EAhy926 endothelial cells. APC elicits anti-apoptotic effects requiring PAR1 and EPCR (24). Hun Lee et al found that progesterone could attenuate thrombin-induced blood-brain barrier disruption by blocking the degradation of tight junction proteins and EPCR in mouse brain endothelial cells bEnd.3 (25). All these studies show that EPCR exerts vascular barrier-protective effect, but the role of EPCR in tumor angiogenesis is not clear. Our results revealed that the expression of EPCR is correlated with MVD in gastric cancer tissue. Knockdown of EPCR expression in MGC803 cells could decrease the proliferation, migration and tubule formation of HUVECs in the presence of the MGC803-conditioned medium, with the medium of MGC803 cells treated with PAR1 antibody having the same effect. Furthermore, EPCR knockdown decreased PAR1 activation. These in vitro events may explain the angiogenic activity of EPCR-PAR1signaling in gastric tumor cells. Uchiba et al found that APC activated the MAPK pathway and induced HUVECs proliferation in vitro. In addition, APC activated endothelial nitric oxide synthase via PI3K phosphorylation, leading to protein kinase $\mathrm{G}$ activation, suggesting that APC bound to EPCR may activate the endothelial MAPK pathway through a mechanism similar to that of VEGF (6). Sen et al found APC-mediated activation of PAR1 and p44/42 MAPK in endothelial cell was enhanced by Zinc ions (26). Gramling et al found that APC enhanced endothelial cell motility and MDA-MB-231 breast cancer cells migration by activating ERK1/2, Akt and NF- $\kappa$ B, but not the JNK pathway (12). The present study showed that the phosphorylation level of ERK1/2 and AKT (S473 and T308) is decreased in HUVECs cultured with the tumor-conditioned medium of MGC803 gastric cancer cells treated with PAR1 antibody or EPCR siRNA. However, further studies are required to investigate EPCR expressed on the tumor cell is how to regulate ERK1/2 and AKT pathway of endothelial cell; whether the role of EPCR is dependent on some pro-angiogenic factors, such as VEGF. 
In conclusion, EPCR exhibits a stimulatory effect on tumor angiogenesis in the human gastric cancer cell line MGC 803 by activating ERK1/2 and AKT, and this effect of EPCR requires PAR1 activation.

\section{Acknowledgements}

Not applicable.

\section{Funding}

This study was supported by the National Natural Science Foundation of China (grant no. 81101493), a General Financial Grant from the China Postdoctoral Science Foundation (grant no. 2014M561713), the Jiangsu Undergraduate Training Program for Innovation and Entrepreneurship (grant no. 201610313008Z) and the Dean Special Foundation of Xuzhou Medical University (grant no. 2012KJZ07).

\section{Availability of data and materials}

The datasets used and/or analyzed during the current study are available from the corresponding author on reasonable request.

\section{Authors' contributions}

PZ designed the study. QW performed the experiments and drafted the manuscript. YT, TW, HY, XW and HM performed the tissue collection, cell culture and data analysis. All authors read and approved the final manuscript.

\section{Ethics approval and consent to participate}

The study protocol was approved by the Medical Ethics Committee at the Affiliated Hospital of Xuzhou Medical University (approval no. xyfylw2012002).

\section{Consent for publication}

Not applicable.

\section{Competing interests}

The authors declare that they have no competing interests.

\section{References}

1. Liu CC, Shen Z, Kung HF and Lin MC: Cancer gene therapy targeting angiogenesis: An updated review. World J Gastroenterol 12: 6941-6948, 2006.

2. Thiyagarajan M, Cheng T and Zlokovic BV: Endothelial cell protein C receptor: Role beyond endothelium? Circ Res 100: 155-157, 2007

3. Crawley JT: Multiple roles of the endothelial cell protein $\mathrm{C}$ receptor. J Thromb Haemost 5: 1813-1816, 2007.

4. Mohan Rao LV, Esmon CT and Pendurthi UR: Endothelial cell protein $\mathrm{C}$ receptor: A multiliganded and multifunctional receptor. Blood 124: 1553-1562, 2014.

5. Riewald M, Petrovan RJ, Donner A and Ruf W: Activated protein $\mathrm{C}$ signals through the thrombin receptor PAR1 in endothelial cells. J Endotoxin Res 9: 317-321, 2003.

6. Uchiba M, Okajima K, Oike Y, Ito Y, Fukudome K, Isobe $\mathrm{H}$ and Suda T: Activated protein C induces endothelial cell proliferation by mitogen-activated protein kinase activation in vitro and angiogenesis in vivo. Circ Res 95: 34-41, 2004.
7. Shua F, Kobayashia H, Fukudomeb K, Tsuneyoshib N, Kimotob M and Teraoa T: Activated protein C suppresses tissue factor expression on U937 cells in the endothelial protein $\mathrm{C}$ receptor-dependent manner. FEBS Lett 477: 208-212, 2000.

8. Keshava S, Sahoo S, Tucker TA, Idell S, Rao LV and Pendurthi UR: Endothelial cell protein $\mathrm{C}$ receptor opposes mesothelioma growth driven by tissue factor. Cancer Res 73: 3963-3973, 2013.

9. Ducros E, Mirshahi S, Azzazene D, Camilleri-Broët S, Mery E, Al Farsi H, Althawadi H, Besbess S, Chidiac J, Pujade-Lauraine E, et al: Endothelial protein C receptor expressed by ovarian cancer cells as a possible biomarker of cancer onset. Int J Oncol 41: 433-440, 2012.

10. Antón I, Molina E, Luis-Ravelo D, Zandueta C, Valencia K, Ormazabal C, Martínez-Canarias S, Perurena N, Pajares MJ, Agorreta $\mathrm{J}$, et al: Receptor of activated protein $\mathrm{C}$ promotes metastasis and correlates with clinical outcome in lung adenocarcinoma. Am J Respir Crit Care Med 186: 96-105, 2012.

11. Heng W, Mu CY, Chen C, Huang JA and Wang ZY: Endothelial cell protein $C$ receptor (EPCR) is expressed by lung carcinoma and correlated with clinical parameters. Clin Lab 59: 375-380, 2013.

12. Gramling MW, Beaulieu LM and Church FC: Activated protein C enhances cell motility of endothelial cells and MDA-MB-231 breast cancer cells by intracellular signal transduction. Exp Cell Res 316: 314-328, 2010.

13. Perurena N, Zandueta C, Martínez-Canarias S, Moreno $\mathrm{H}$, Vicent S, Almeida AS, Guruceaga E, Gomis RR, Santisteban M, Egeblad M, et al: EPCR promotes breast cancer progression by altering SPOCK1/testican 1-mediated 3D growth. J Hematol Oncol 10: 23, 2017.

14. Wang Q, Liu Q, Wang T, Yang H, Han Z and Zhang P: Endothelial cell protein $\mathrm{C}$ receptor promotes MGC803 gastric cancer cells proliferation and migration by activating ERK1/2. Med Oncol 32: 162, 2015

15. Jang HK, Kin BS, Han J, Yoon JK, Lee JR, Jeong GJ and Shin JY: Therapeutic angiogenesis using tumor cell-conditioned medium. Biotechnol Prog 32: 456-464, 2016.

16. Xu LN, Xu BN, Cai J, Yang JB and Lin N: Tumor-associated fibroblast-conditioned medium promotes tumor cell proliferation and angiogenesis. Genet Mol Res 12: 5863-5871, 2013.

17. Zhang T and Jiang CL: Tumor conditioned medium regulates the proliferation, adhesion and migration of human umbilical vein endothelial cells. Sheng Li Xue Bao 63: 256-260, 2011 (In Chinese).

18. Liu T, Jabbes M, Nedrow-Byers JR, Wu LY, Bryan JN and Berkman CE: Detection of prostate-specific membrane antigen on HUVECs in response to breast tumor-conditioned medium. Int J Oncol 38: 1349-1355, 2011.

19. Koizumi S, Gu C, Amano S, Yamamoto S, Ihara H, Tokuyama T and Namba H: Migration of mouse-induced pluripotent stem cells to glioma-conditioned medium is mediated by tumor-associated specific growth factors. Oncol Lett 2: 283-288, 2011.

20. Peng Y, Li J and Geng M: The glycan profile of endothelial cells in the present of tumor-conditioned medium and potential roles of beta-1,6-GlcNAc branching on HUVEC conformation. Mol Cell Biochem 340: 143-152, 2010.

21. Falahat R, Wiranowska M, Gallant ND, Toomey R, Hill R and Alcantar N: A cell ELISA for the quantification of MUC1 mucin (CD227) expressed by cancer cells of epithelial and neuroectodermal origin. Cell Immunol 298: 96-103, 2015.

22. Niessen F, Furlan-Freguia C, Fernández JA, Mosnier LO, CastellinoFJ, Weiler H, Rosen H, Griffin JH and RufW: Endogenous EPCR/aPC-PAR1 signaling prevents inflammation-induced vascular leakage and lethality. Blood 113: 2859-2866, 2009.

23. Sundaram J, Keshava S, Gopalakrishnan R, Esmon CT, Pendurthi UR and Rao LV: Factor VIIa binding to endothelial cell protein $\mathrm{C}$ receptor protects vascular barrier integrity in vivo. J Thromb Haemost 12: 690-700, 2014.

24. Mosnier LO and Griffin JH: Inhibition of staurosporine-induced apoptosis of endothelial cells by activated protein $\mathrm{C}$ requires protease-activated receptor-1 and endothelial cell protein $\mathrm{C}$ receptor. Biochem J 373: 65-70, 2003.

25. Hun Lee J, Won S and Stein DG: Progesterone attenuates thrombin-induced endothelial barrier disruption in the brain endothelial cell line bEnd.3: The role of tight junction proteins and the endothelial protein C receptor. Brain Res 1613: 73-80, 2015.

26. Sen P, Sahoo S, Pendurthi UR and Rao LV: Zinc modulates the interaction of protein $\mathrm{C}$ and activated protein $\mathrm{C}$ with endothelial cell protein C receptor. J Biol Chem 285: 20410-20420, 2010.

This work is licensed under a Creative Commons Attribution-NonCommercial-NoDerivatives 4.0 International (CC BY-NC-ND 4.0) License. 